\title{
Randomised prospective masked study comparing patient comfort following the instillation of topical proxymetacaine and amethocaine
}

\author{
Taher Shafi, Peter Koay
}

\begin{abstract}
Aims-To evaluate the claim that topical proxymetacaine produces little or no discomfort on instillation by comparing it against topical amethocaine.

Methods-This randomised, masked, double blind, prospective study involved 53 consecutive patients. Each patient received one drop of amethocaine $0.5 \%$ in one eye and one drop of proxymetacaine $0.5 \%$ in the other. The duration of the stinging sensation and degree of discomfort (using descriptive and a linear analogue method) for each topical anaesthetic was assessed.

Results-The mean duration of stinging was 3.2 seconds for proxymetacaine and 22.1 seconds for amethocaine the difference being statistical significant (p $<0.001)$. The mean descriptive discomfort score was 2.6 for proxymetacaine and $\mathbf{1 4 . 2}$ for amethocaine $(p=0.01)$. The mean linear analogue score was $\mathbf{5 . 8}$ for proxymetacaine and 35.6 for amethocaine $(p<0.001)$. Proxymetacaine had a successful tonometry rate of $93 \%$ and amethocaine $98 \%$.

Conclusion-This clinical study confirms that proxymetacaine is more comfortable on instillation than amethocaine. Minimising unnecessary patient discomfort is not only ideal, but encourages cooperation in apprehensive patients especially in children.

(Br f Ophthalmol 1998;82:1285-1287)
\end{abstract}

Topical ophthalmic anaesthetic agents available since the $1930 \mathrm{~s}^{1}$ are among the commonest eye drops used in diagnostic and therapeutic ophthalmology. Most topical anaesthetics cause stinging and discomfort on instillation, as evidenced by lacrimation and blepharospasm. It is easy to disregard these uncomfortable effects as "necessary" without considering alternatives. Among the various topical anaesthetics, it has been suggested that proxymetacaine produces little or no discomfort on instillation. $^{2}$ The aim of our study was to evaluate such a claim in a clinical setting, by comparing it against an established anaesthetic agent such as amethocaine. ${ }^{3}$

Hospital, Darlington

DL3 6HX

T Shafi

P Koay

Correspondence to: Dr Shafi.

Accepted for publication 12 May 1998 outpatient department requiring tonometry. The exclusion criterion are as follows: oph- thalmic conditions that alter corneal sensation, ocular inflammatory conditions, infections, uveitis, scleritis, blepharitis, corneal ulcerations, corneal dystrophies, patients undergoing anti-inflammatory treatments, acute glaucoma, foreign bodies, photophobia, and any pathology that can cause ocular irritation. Informed consent was obtained from participating patients.

Amethocaine $0.5 \%$ and proxymetacaine $0.5 \%$ eye drops were randomly assigned to either " $\mathrm{A}$ " or " $\mathrm{B}$ ". This assignment was unknown to the researchers until the study was completed and the code revealed. A recruited nurse, independent of the examiner instilled either " $A$ " or " $B$ " into the middle portion of the lower conjunctival fornix of one eye. On instillation of the drop the patient was asked to look up and the lower eyelid was gently retracted from the globe. For both drops minims, sterile, single use, disposable applicators was used. Each minim applicator contains 0.5 $\mathrm{ml}$ of the anaesthetic drop. One drop $(50 \mu \mathrm{l})$ was instilled into the lower fornix. The duration of the pain was recorded. After 1 minute the tonometry was performed to assess the anaesthetic effect. The opposite eye was then instilled with the other eye drop. For example, if the right eye received " $A$ " then the left eye would receive "B". Each patient received one drop of amethocaine $0.5 \%$ in one eye and proxymetacaine $0.5 \%$ in the other.

The duration of the stinging sensation immediately after the instillation of " $A$ " or " $B$ " was measured. The severity of discomfort following the eye drops was assessed using both a descriptive method and a linear analogue method. In the descriptive method, patients assigned the sensation of discomfort into categories - "no pain", "mild pain", "moderate pain", "severe pain", and "very severe pain". These five categories were arbitrarily scored from 0 to 4 respectively. This was followed by assessments using a $100 \mathrm{~mm}$ unmarked linear analogue discomfort scale. ${ }^{4}$ Subjects were asked to score the severity of their discomfort on the linear analogue scale ranging from "no pain" to "very severe pain".

Patients' preference of either drop " $A$ " or " $B$ " or lack of preference was noted. To confirm the proper instillation of the anaesthetic agents, scheduled tonometry using a Tonopen was performed 5 minutes after drop instillation, providing evidence of satisfactory anaesthetic effect. Tonometry was regarded as a success if it was easily performed and without patient discomfort. Tonometry was regarded as unsuccessful if 


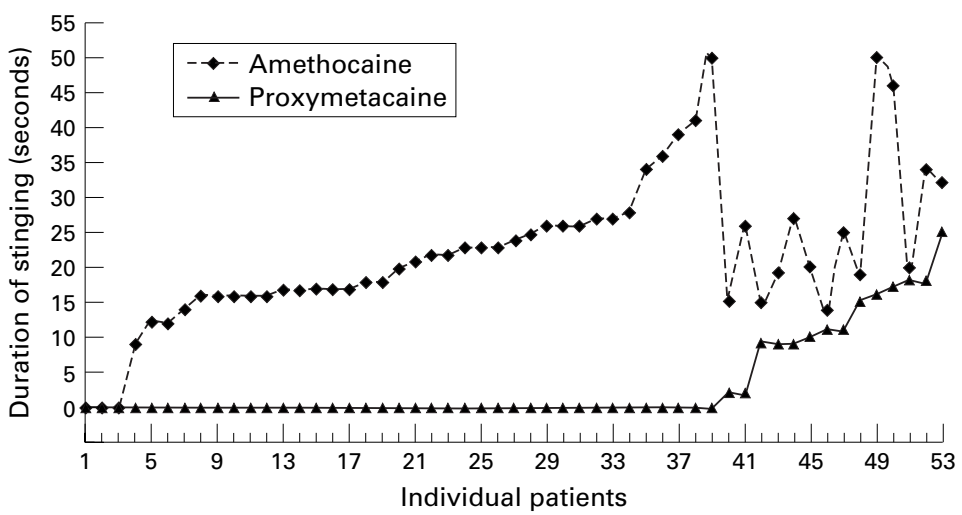

Figure 1 Duration of stinging sensation reported by individual patients receiving topical amethocaine and proxymetacaine.

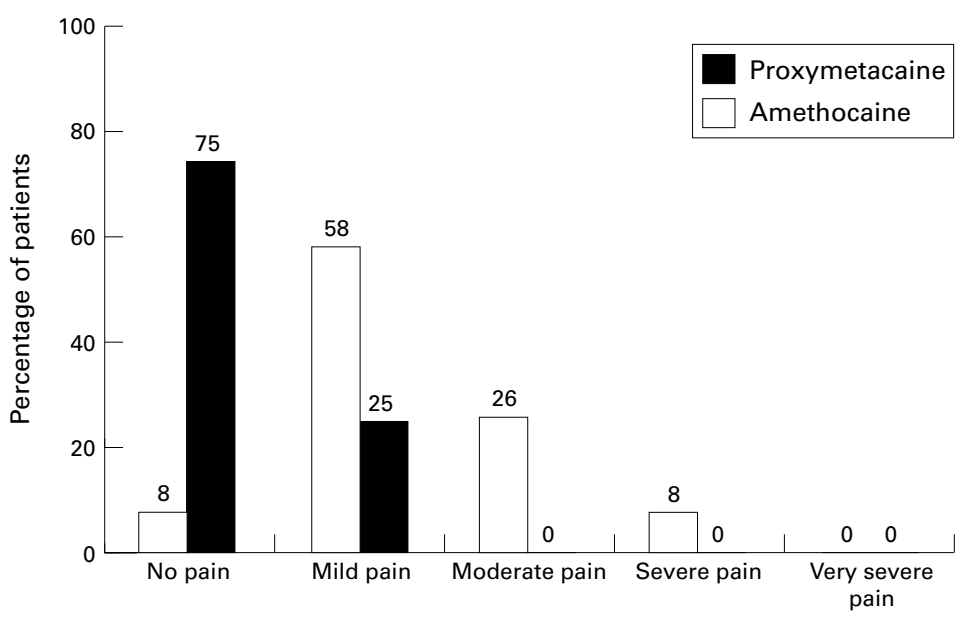

Figure 2 Percentage of patients experiencing "no pain", "mild pain”, "moderate pain", and "severe pain" as expressed on the descriptive discomfort scale.

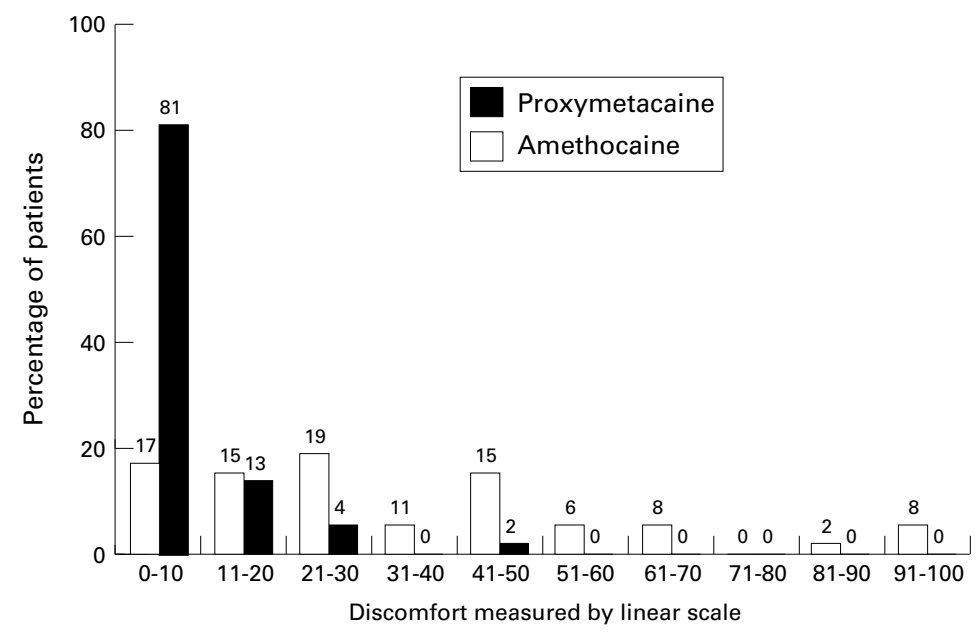

Figure 3 Percentage of patients experiencing discomfort measured by the $100 \mathrm{~mm}$ unmarked linear analogue scale.

the patient felt uncomfortable. Another drop of the same anaesthetic agent was instilled should this happen and the procedure was repeated. Any adverse reactions were documented.

\section{Results}

In the sample of 53 consecutive patients, 17 were males and 36 females. The mean age was 64.7 years (SD 19.5, range 19-87 years).

Figure 1 shows the distribution of the duration of stinging in individual patients. The mean duration of stinging for proxymetacaine was 3.2 (SD 6.3) seconds compared with the mean of 22.1 (10.7) seconds of stinging for amethocaine. There was statistical significance ( $p<0.001)$ in the difference of the above means. The mean duration of stinging overall in both groups was 12.7 (12.9) seconds.

The percentage of patients who felt that the duration of stinging was longer using amethocaine compared with proxymetacaine was $94.3 \%(n=50)$. Only one patient $(1.3 \%)$ felt that duration of stinging was longer using proxymetacaine. Two patients $(2.6 \%)$ felt that there was no difference in the duration of the stinging between proxymetacaine and amethocaine.

Figure 2 shows the percentage of patients experiencing "no pain", "mild pain", "moderate pain", "severe pain", and "very severe pain" on the descriptive discomfort scale for eyes receiving amethocaine and proxymetacaine. Using the scoring described above, the mean descriptive discomfort score was 2.6 for proxymetacaine and $\mathbf{1 4 . 2}$ for amethocaine, the difference being statistically significant $(p=$ 0.01 ) using the Wilcoxon rank sum test.

Figure 3 shows the distribution of discomfort along the unmarked linear analogue scale of length $100 \mathrm{~mm}$ for eyes receiving amethocaine and proxymetacaine. The mean linear analogue score was $5.8(0.9) \mathrm{mm}$ for proxymetacaine and $35.6(2.6) \mathrm{mm}$ for amethocaine. The differences between the means was statistically significant at $(\mathrm{p}<0.001)$ using the $t$ test.

Eighty nine per cent of patients $(n=47)$ preferred proxymetacaine while only one patient preferred amethocaine. Nine per cent $(n=5)$ felt that there was no difference in either using proxymetacaine or amethocaine.

Proxymetacaine had a successful tonometry rate of $93 \%(n=49)$ and unsuccessful tonometry of $7 \%(n=4)$. Amethocaine had a success rate of $98 \%(n=52)$ and unsuccessful of $2 \%(n=1)$. The differences between the success rates between proxymetacaine and amethocaine were not statistically significant $(\mathrm{p}=0.08)$.

When tonometry was regarded as unsuccessful, a further one drop of the same anaesthetic agent was instilled. Tonometry was then subsequently comfortable in all patients. No adverse reactions or side effects were observed.

\section{Discussion}

Topical anaesthetics act by producing reversible blockade of the sensory nerve endings ${ }^{5}$ and blocking action potentials responsible for nerve conduction at the cell membrane level. ${ }^{67}$ Amethocaine (a para-aminobenzoic acid) and proxymetacaine (a benzoate ester) are naturally weak bases and are slightly soluble in unionised forms. ${ }^{8}$ To improve the solubility, commercial anaesthetic agents are usually made acidic as soluble hydrochlorides. ${ }^{9}$ This allows for a longer and more stable shelf life.

The $\mathrm{pH}$ of anaesthetic agents affects the degree of comfort on instillation. Amethocaine has a $\mathrm{pH}$ of 4.54 and proxymetacaine a $\mathrm{pH}$ of 4.64. ${ }^{3}$ This probably explains why amethocaine (being more acidic) "stings" more than proxymetacaine. Although tears can dilute and 
buffer topical anaesthetic agents, this effect is not sufficient to cope with the immediate relatively large volume of anaesthetic instilled, resulting in discomfort on instillation.

This study shows that proxymetacaine is more comfortable than amethocaine for the duration of stinging (Fig 1) and for the severity of the discomfort on both descriptive (Fig 2) and linear analogue assessments (Fig 3). When one drop of anaesthetic agent was used, successful tonometry was $93 \%$ using proxymetacaine compared with $98 \%$ using amethocaine. Although these observations were not statistically significant, there is a possibility that proxymetacaine may be less efficient as an anaesthetic agent than amethocaine. The reason for the difference may be due to the difference in the $\mathrm{pH}$ between the two anaesthetic agents, amethocaine being more acidic. ${ }^{310}$

The current retail price in the United Kingdom for a box of $200.5 \mathrm{ml}$ minims of proxymetacaine is $£ 6.95$ and $£ 5.75$ for amethocaine. ${ }^{9}$ Although there is a 6 pence price difference per unit dispensed, the retail prices can be affected by bulk purchase.

\section{Conclusion}

This clinical study indicates that proxymetacaine is more comfortable on instillation than amethocaine. Minimising unnecessary patient discomfort is not just an ideal but is likely to encourage cooperation in apprehensive patients. In the paediatric ophthalmology, the use of eye drops which "hurt" encourages mistrust, hampering subsequent cooperation which can limit the success of the visit.

We wish to acknowledge Lin Cordey, Ann Holden, and Sara Davies for their contribution to this study.

1 Koller C. Preliminary report on local anaesthetics of the eye. Arch Ophthalmol 1934;12:473-4.

2 Bartlett JD, Jaanus SD. Local anesthetics. In: Bartlett JD, aanus SD, eds. Clinical ocular pharmacology. 2nd ed. London: Butterworth-Heinemann, 1989;4:149-62.

3 Bartfield JM, Holmes TJ, Raccio-Robak N. A comparison of proparacaine and tetracaine eye anaesthetics. Acad Emerg Med 1994;1:364-7.

4 Scott J, Huskisson EC. Graphic representation of pain. Pain 1976;2:175-84.

5 Webster RB. Local anaesthetics for ophthalmic use. Aust $\mathcal{F}$ Optom 1974;57:399-401.

6 Catterall W, Mackie K. Local anaesthetics. In: Hardman JG, Limdind LE, eds. The pharmalogical basis of therapeutics. Goodman \& Gilman. 9th ed. Oxford: Pergamon Press, 1996;15:331-47.

7 Wilson RP. Anesthesia. In: Spaeth GL, eds. Ophthalmic surgery principles and practice. 2nd ed. Philadelphis: Saunders 1990;5:75-99.

8 Ritchie JM, Greene NM. Local anaesthetics. In: Gilman basis of therapeutics. 8th ed. Elmsford: Paragon Press, 1990; 311-31.

9 Mehta DK, Martin J, Townsend-Smith SM, et al, eds. British national formulary No 37. London: BMA, September 1997:451.

10 Christoph RA, Buchanan L, Beglia K, et al. Pain reduction in local anesthetic administration through $\mathrm{pH}$ buffering. Ann Emerg Med 1988;17:117-20. 